\title{
Ictal Swearing as a Lateralizing Value for the Dominant Hemisphere in Temporal Lobe Epilepsy
}

Case Report

Journal of Epilepsy Research pISSN 2233-6249 / eISSN 2233-6257

Received May 29, 2021

Revised June 18, 2021

Accepted June 22, 2021

Corresponding author:

Dae Lim Koo, MD, PhD

Department of Neurology, Seoul

Metropolitan Government Seoul National

University Boramae Medical Center and

Seoul National University College of

Medicine, 20 Boramae-ro 5-gil, Dongjak-gu,

Seoul 07061, Korea

Tel. $+82-2-870-2473$

Fax. +82-2-831-0714

E-mail; koodaelim@gmail.com, koodaelim@snu.ac.kr

\author{
Woohee Ju, MD, Jong-Su Kim, MD, Yangmi Park, MD, Dae Lim Koo, MD, PhD, \\ Hyunwoo Nam, MD, PhD
}

Department of Neurology, Seoul Metropolitan Government Seoul National University Boramae Medical Center and Seoul National University College of Medicine, Seoul, Korea

Ictal swearing, as an epileptic manifestation, has rarely been reported. Despite its poor localization value and unclear mechanism, several previous studies have reported that it frequently originates from the temporal lobe and more often from the non-dominant hemisphere. Herein, we report a case of a 41-year-old right-handed man with a history of stereotypical manifestation of ictal swearing with a left (dominant) hemisphere origin, confirmed by video electroencephalography monitoring. Reasonable suspicion that repetitive swearing could be a manifestation of seizures is important for clinicians not to misdiagnose the disease. (2021;11:110-111)

Key words: Ictal swearing, Temporal lobe epilepsy, Seizures

\section{Introduction}

Ictal swearing, a culturally taboo offensive utterance ${ }^{1}$ is a rare manifestation in epilepsy patients, ${ }^{2-4}$ and its pathophysiology and localizing value of ictal swearing are unclear, although a variety of automatisms, including ictal speech, are lateralizing signs for the non-dominant hemisphere. ${ }^{1-5}$ Herein, we report a patient with ictal swearing, with ictal discharges on the temporal lobe of the dominant hemisphere, confirmed by video electroencephalography (EEG) monitoring.

\section{Case Report}

A 41-year-old right-handed man presented with episodes of ictal swearing with loss of consciousness lasting for approximately $1 \mathrm{mi}-$ nute and occurring mostly during daytime naps at his workplace. These stereotypic episodes had started 2 months before his visit to the epilepsy clinic. He had no other significant medical history, including perinatal complications, febrile convulsions, central nervous system infections or trauma, or a family history of epilepsy.

During the 3 days of scalp video-EEG monitoring (VEM), three episodes of stereotypic seizures were recorded. Each ictal episode lasted for approximately 1 minute, and the longest was approximately 2 minutes. The seizures started with sudden awakening, followed by stereotypical swearing with "ssibal" ("fuck" in Korean) (Supplementary Videos 1, 2). Ictal discharges of the VEM revealed that rhythmic theta activities started from the left temporal region and propagated to the left hemisphere with semi-rhythmic delta activities (Fig. 1A). T2 fluid-inversion recovery (FLAIR) brain magnetic resonance imaging (MRI) showed high signal intensity in the left amygdala (Fig. 1B). The patient was diagnosed with epilepsy but was seizure-free during the 8-month follow-up period after taking oxcarbazepine at 1,200 mg/day.

\section{Discussion}

Ictal swearing is more common among males, with lateralization to the non-dominant hemisphere. ${ }^{2,4,6}$ However, Massot-Tarrús et al. ${ }^{1}$ reported that five out of nine subjects were women, and the lateralizing value of the seizures was unclear. At the onset of swearing, ictal EEG activities were localized to the mesial temporal or orbitofrontal regions in all patients, regardless of the hemispheric side, ${ }^{1,5}$ which was consistent with our case. Although ictal speech has a lateralizing value for non-dominant hemispheric seizures, our case with ictal 

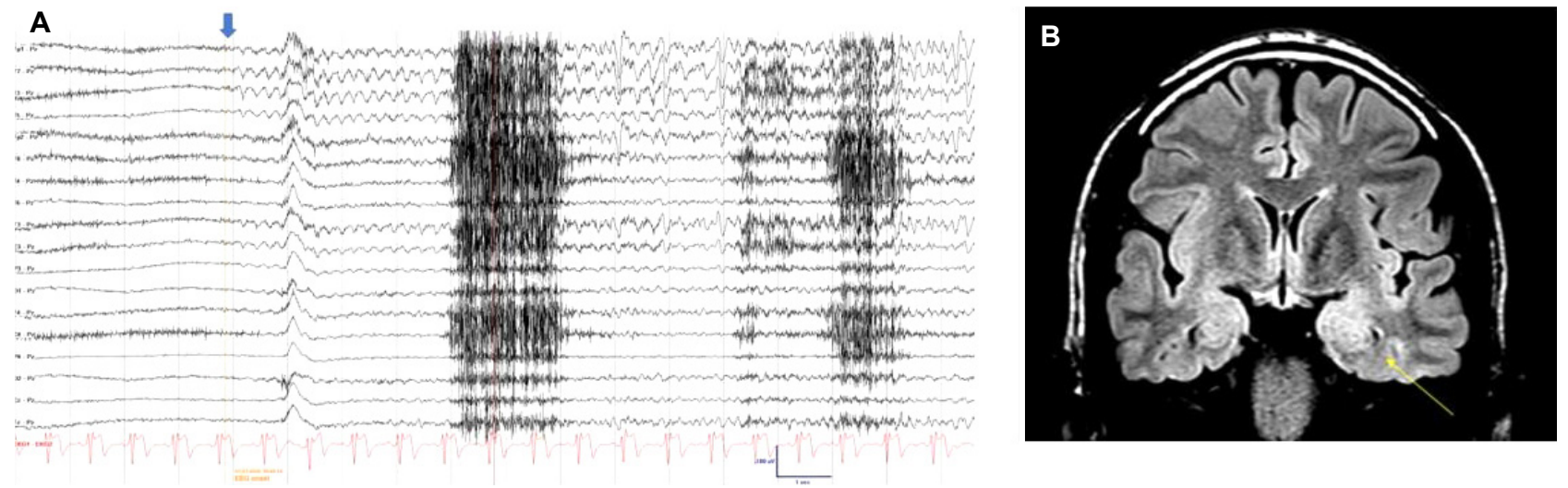

Figure 1. (A) Ictal discharge started with theta activities from the left temporal area (blue arrow), followed by rhythmic delta activities in the left hemisphere. (B) Coronal sections of a T2 fluid-inversion recovery magnetic resonance imaging scan revealed a high signal intensity in the left amygdala (yellow arrow).

swearing revealed an epileptic focus in the dominant hemisphere.

Ictal swearing is a verbal automatism associated with negative emotion, ${ }^{3}$ whereas ictal speech is characterized by comprehensible speech. ${ }^{4}$ This implies a different neurophysiological process between ictal swearing and ictal speech. ${ }^{2,5}$ Ictal speech results from activation or inhibition of language cortical function, whereas the hypothesized mechanism of ictal swearing is the dysregulation of emotional and behavioral function. ${ }^{4}$ In this context, limbic frontal-temporal networks can produce ictal swearing. ${ }^{1,3}$ The orbitofrontal cortex (OFC) receives inputs from all sensory modalities, including the amygdala, hippocampus, cingulate cortex, and projects back to the temporal lobe area. ${ }^{3,4}$ The inhibitory connection from the OFC to the amygdala regulates negative emotions and related behaviors. ${ }^{1,3}$

In our patient, FLAIR MRI showed a high signal intensity in the left amygdala, which is thought to be a seizure-related change. Despite the lack of further imaging studies, including functional MRI, considering all the findings of symptomatic semiology, ictal EEG discharges, and MRI scans, the amygdala might be an important candidate for the pathophysiological mechanism of ictal swearing. Our case provides evidence for a possible neurophysiological process of ictal swearing. Further neuroimaging studies, including functional MRI and positron emission tomography, should be conducted to clarify the underlying mechanism.

To the best of our knowledge, this is the first case report of repetitive ictal swearing originating from the dominant hemisphere, which was concordantly localized by VEM recording and MRI findings. Our case showed that a detailed history of the presence of stereotypy of ictal events could improve diagnostic accuracy for epilepsy and reduce the probability of misdiagnosis of vocal tics or violent profanity.

\section{Conflict of Interest}

The authors declare that they have no conflicts of interest.

\section{Supplementary materials}

Supplementary data to this article can be found online at https://www.j-epilepsy.org/.

\section{References}

1. Massot-Tarrús A, Mousavi SR, Dove $C$, et al. Coprolalia as a manifestation of epileptic seizures. Epilepsy Behav 2016;60:99-106.

2. Birca V, Tayah T, Saint-Hilaire JM, Nguyen DK. Ictal swearing: a case series and review. Epileptic Disord 2013;15:377-81.

3. Zhang $H$, Wang $D$, Ren L, Fan $X$, Shan $Y$, Zhao G. Ictal swearing network confirmed by stereoencephalography: a case report. Acta Neurochir (Wien) 2019;161:2499-503.

4. Zhang $X$, Zhang $G$, Ma $K$, et al. A case of right orbitofrontal epilepsy featuring ictal swearing. J Neurol Sci 2019;397:1-3.

5. Dolatshahi M, Yankovsky A. Ictal forced repetitive swearing in frontal lobe epilepsy: case report and review of the literature. J Epilepto/ 2014; 22:113-9.

6. Quevedo-Diaz M, Campo AT, Vila-Vidal M, Principe A, Ley M, Rocamora R. Ictal spitting in non-dominant temporal lobe epilepsy: an anatomo-electrophysiological correlation. Epileptic Disord 2018;20:139-45. 
Supplementary Video 1. The seizure started with ictal swearing, followed by fumbling of the left hand and immobile posturing of the right arm. Ictal oroalimentary automatism was observed during the ictal event, followed by postictal nose wiping. 
Supplementary Video 2. The seizure began with ictal swearing, and the automatic behavior of lip-smacking occurred during the ictal event. 